\title{
Usage of lipid-lowering drugs such as atorvastatin and rosuvastatin in the management of hyperlipidemia in multiple vessel wall block patients
}

\author{
Sivaraj R. ${ }^{*}$, Umarani S. ${ }^{2}$
}

${ }^{1}$ Department of Pharmacology, Aarupadaiveedu Medical

College, and Hospital,

Kirumampakkam, Puducherry, India

${ }^{2}$ Department of Anatomy, Rajah Muthiah Medical College and Hospital, Annamalai University, Annamalainagar, Tamil Nadu, India

Received: 31 October 2017 Accepted: 24 November 2017

*Correspondence to:

Dr. Sivaraj R.

Email: drrsivaraj@gmail.com

Copyright: () the author(s), publisher and licensee Medip Academy. This is an openaccess article distributed under the terms of the Creative Commons Attribution NonCommercial License, which permits unrestricted noncommercial use, distribution, and reproduction in any medium, provided the original work is properly cited.

\begin{abstract}
Background: Multi-vessel coronary artery disease (CAD) is a disease stage in which at least two or three of the epicardial coronary arteries is involved with atherosclerosis of significant severity. The multi-vessel disease is often associated with a higher burden of comorbidities, left ventricular dysfunction, and cardiovascular risk. Dyslipidaemia is the commonest cause of the blood vessel diseases and their incidence has been rising all over the world thereby increasing the morbidity and mortality due to cardiovascular diseases. Dyslipidaemia is also one of the component of metabolic syndrome along with another group of cardiovascular risk factors such as high blood pressure (BP), abdominal obesity, and insulin intolerance, whose concurrent appearance increases the risk of atherosclerotic cardiovascular disease. To compare the efficacy of atorvastatin and rosuvastatin in the management of hyperlipidaemia. To compare the doserelated efficacy of statins on lipid goal achievement in patients with hyperlipidaemia.
\end{abstract}

Methods: This prospective, randomized, single-blinded interventional study was conducted for a period of 1 year [2014-1015] in medicine OPD in Rajah Muthiah Medical College and Hospital. A total number of 100 patients with diagnosed multiple vessel blocks were included in this study. Among them, 50 patients were randomized to group A. 50 patients were selected to group B. 50 patients of the group A are treated with Atorvastatin up $10 \mathrm{mg}$ once daily for 8 weeks. 50 patients of group B were treated with Rosuvastatin up to $10 \mathrm{mg} /$ day. For 8 weeks the results were analysed accordingly.

Results: Even though both rosuvastatin $10 \mathrm{mg} /$ day and atorvastatin $10 \mathrm{mg} /$ day produced a reduction in total cholesterol $(\mathrm{p}<0.001)$ significantly, rosuvastatin produced a reduction in LDL levels $(\mathrm{p}<0.001)$ more significantly than atorvastatin $10 \mathrm{mg} /$ day.

Conclusions: Rosuvastatin produces a greater reduction in serum LDL-C levels and should, therefore, be preferred over atorvastatin. Both the treatment regimens significantly decreased TC, TG, LDL C, VLDL C, but the reduction was more and statistically significant in Rosuvastatin when compared with atorvastatintreated group at the end of 8 weeks.

Keywords: Atorvastatin, Coronary angioplasty LDL, Dyslipidemia, HDL, Multiple vessel blocks, Rosuvastatin

\section{INTRODUCTION}

Patients with diabetes mellitus have a higher risk of cardiovascular mortality than the general population. This increase in risk is believed to be related to accelerated coronary atherosclerosis, a higher frequency of complications of the coronary disease, including myocardial infarction (MI), as well as a higher likelihood of mortality resulting from those complications. ${ }^{1}$ Although only $5 \%$ of the population have diabetes; $13 \%$ to $25 \%$ of the patients undergoing coronary revascularization procedures have diabetes. Patients with diabetes experience higher perioperative as well as midterm mortality rates compared with nondiabetics undergoing 
coronary artery bypass grafting (CABG). However, patients with diabetes have also been shown to have less favorable long-term survival after percutaneous coronary intervention (PCI). Thus, for patients with diabetes requiring revascularization, the choice of procedure has provoked much controversy. ${ }^{2}$ The bypass angioplasty revascularization investigation (BARI) trial demonstrated that, for patients with treated diabetes mellitus and multivessel coronary artery disease (MVD) who were candidates for either $\mathrm{CABG}$ or PCI, initial CABG was associated with a markedly lower five-year mortality rate relative to initial PCI ( $19.4 \%$ vs. $34.5 \%$, respectively, $\mathrm{p}=$ 0.003 ) This result triggered a national heart, lung and blood institute (NHLBI) clinical alert recommending bypass surgery in this patient group. ${ }^{3}$ Dyslipidemia occurs due to a disturbance in the lipid parameters like Total Cholesterol, LDL-C, VLDL, TGs, and HDL-C. Combined or mixed hyperlipidemia (CHL) is a lipid disorder characterized by increased low-density lipoprotein cholesterol (LDL-C), elevated triglycerides (TGs) and decreased high-density lipoprotein cholesterol (HDL-C) which is more common in patients with type 2 diabetes mellitus. It has been estimated that almost every other adult in the United States has abnormal cholesterol values and every third person has elevated low-density lipoprotein cholesterol (LDL-C) levels. ${ }^{4}$ Not only is the treatment of hyperlipidemia associated with improved outcomes in patients with these diseases, but also the lipidlowering the most powerful intervention in primary prevention. Statins are the first-line therapy for treating high lipid levels. In addition to the numeric reduction.in lipid levels, they significantly reduce vascular events and all-cause mortality through their pleiotropic effects. ${ }^{5}$ It has already been proved that statins have antioxidant, antiinflammatory effects and antithrombotic properties that add to their clinical utility. They improve endothelial dysfunction and reduce the growth of atherosclerotic plaque Available evidence does not strongly suggest a clear clinical benefit of other lipid-lowering agents in such situations. All of the available statins have small differences in terms of pharmacokinetics and pharmacodynamics and hence clinical efficacy and side effects profile. $^{6}$ The treatment of hyperlipidemia in coronary heart disease is of two types.

1. Primary Prevention is to treat Hyperlipidemias in a patient who is prone to develop Coronary Heart Disease.

2. Secondary prevention is to treat patients who already have the Coronary Heart Disease.

The drug treatment of hyperlipidemias includes fabric acid derivatives, cholestyramine Resin, 3-hydroxy 3-methyl glutaryl coenzyme-A (HMG Co-A) reductase inhibitors (statins), estrogens, probucal, fibre, Gugulipid, nicotinic acid, selective estrogen receptor modulators. Nowadays, coronary heart disease is one of the major cause of death and hyperlipidemias are one of the main cause of coronary heart disease. Among all drugs, the statins are widely used in the treatment of hyperlipidemias and to decrease the coronary heart disease. The broad range of significant clinical benefits of statin therapy includes a decrease in major coronary events, coronary revascularization, stroke and transient ischemic attack, death due to CHD and total mortality. ${ }^{7}$ Based on this we undertook this study to compare the efficacy of rosuvastatin and atorvastatin. As there are many patients having CHD this study has been undertaken, i.e. the treatment of hyperlipidemia in CHD patients. $^{8}$

\section{METHODS}

This was a prospective and comparative study, performed in 100 patients attending the cardiology and medicine outpatient department, rajah mutaiah medical college and hospital. All patients men and women aged 30-70 Years with a previous history of Acute Myocardial infarction or Unstable angina $>3$ months but $<20$ months were selected. This study was conducted on patients with lipid levels in the range of Total Cholesterol $<240 \mathrm{mg} / \mathrm{dl}$; Triglycerides $<350 \mathrm{mg} / \mathrm{dl}$, LDL-C $>100 \mathrm{mg} / \mathrm{dl}$ were selected for the study. Before starting the study, the Ethical committee approved the protocol. Written and informed consent was obtained from each patient in the local language.Initially, 100 patients were recruited into the study who met inclusion and exclusion criteria. These patients were selected and placed into two groups.

- Group A was given Atorvastatin 10mg once daily.

- Group B was given Rosuvastatin 10mg once daily, during the whole period of the study.

\section{Inclusion Criteria}

- Adults and geriatrics patients

- Patients who are diagnosed with hyperlipidemia

- Patients who are having an established prescription for hyperlipidemia

- Low Density Lipoprotein-Cholesterol $>100 \mathrm{mg} / \mathrm{dl}$

- Serum creatinine $<1.2 \mathrm{mg} / \mathrm{dl}$

- Normal liver function test

\section{Exclusion criteria}

- Patients with cognitive problems

- Pregnant patients

- Patients with a major disorder of hepatic, GIT or Hemopoietic systems

- Patients who are not willing to participate

- Patients with fluctuating or rapidly deteriorating function

Before starting the study, the risk factors like smoking, hypertension, $\mathrm{H} / \mathrm{o}$ of unstable angina (USA) and H/o myocardial infarction (MI) were noted for each patient. Among the 100 patients in the study 59 patients had previous MI (30 patients in atorvastatin group and 29 patients in rosuvastatin group), 39 patients had unstable angina (20 patients in atorvastatin group and 19 patients in 
rosuvastatin group). The laboratory investigations performed initially were $\mathrm{Hb} \%$, blood sugar, blood urea, Serum creatinine, serum bilirubin and lipid profile (total cholesterol, triglycerides, LDL-C, HDL-C). The ECG was taken, echocardiography records of the patients done at the time of discharge from the previous hospitalization were checked for ventricular dysfunction. On each visit, compliance to treatment was assessed and possible side effects of treatment were recorded. Serum total cholesterol, TG, HDL-C, and LDL-C after 12 hours fast were rechecked at the end of 8 weeks.

\section{Statistical analysis}

Mean \pm SD values were calculated for each variable. Demographic details were summarized for all subjects using descriptive statistics. Pairwise comparisons within the groups and between the two treatments were tested for statistical significance using the paired and unpaired Student $t$ test respectively. Statistical significance was at $\mathrm{P}<0.05$. All statistical tests were processed using graph pad prism software, Version 5.0

\section{RESULTS}

Essential hypertension, was found to be more in group B (47) when compared to group A (43) (Table 1). DM was found to be more number in the group a (48) when compared to group B (46). The congestive cardiac failure rate was found to be very less or rare in both the groups. Unstable angina was found to be 7 in group A group and more 11 in group B.

Table 1: Basic underlying disease components.

\begin{tabular}{|lll|}
\hline Underlying disease & $\begin{array}{l}\text { Group A } \\
(\boldsymbol{n}=\mathbf{5 0})\end{array}$ & $\begin{array}{l}\text { Group B } \\
(\boldsymbol{n}=\mathbf{5 0})\end{array}$ \\
\hline Essential hypertension & 43 & 47 \\
\hline Diabetes mellitus & 48 & 46 \\
\hline Congestive cardiac failure & 1 & 0 \\
\hline Unstable angina & 7 & 11 \\
\hline
\end{tabular}

TLC was found to be more in group A $(318 \pm 39.16)$ when compared to group B $(306 \pm 46.44)$ which was around -39 $\%$ which is statically significant (Table 2 ).

Mean triglyceride was found to be more in group A $(298.9 \pm 14.56)$ when compared to group B $(286.4 \pm 11.38)$ which was around $-41 \%$ which is statically significant. HDL was found to be more in group B $(35.04 \pm 8.15)$ when compared to group A $(35.04 \pm 8.15)$ which was around +9 $\%$ which is statically less significant. LDL was found to be more in group A $(233.6 \pm 29.06)$ when compared to group B (212.90 \pm 35.09$)$ which was around $-63 \%$ which is statically significant. VLDL was found to be more in group A $(49.56 \pm 98)$ when compared to group B $(46.99 \pm 74)$ which was around $63 \%$, which is statically significant.

Table 2: Lipid profile levels among the two groups before treatment.

\begin{tabular}{|llllll|}
\hline Groups $[\mathbf{n = 1 0 0}]$ & $\begin{array}{l}\text { Mean TLC } \\
(\mathbf{m g} / \mathbf{d L})\end{array}$ & $\begin{array}{l}\text { Mean TGL } \\
(\mathbf{m g} / \mathbf{d L})\end{array}$ & $\begin{array}{l}\text { Mean HDL } \\
(\mathbf{m g} / \mathbf{d L})\end{array}$ & $\begin{array}{l}\text { Mean LDL } \\
(\mathbf{m g} / \mathbf{d L})\end{array}$ & $\begin{array}{l}\text { Mean VLDL } \\
(\mathbf{m g} / \mathbf{d L})\end{array}$ \\
\hline Atorvastatin $(\mathrm{n}=50)$ & $318 \pm 39.16$ & $298.9 \pm 14.56$ & $35.04 \pm 8.15$ & $233.6 \pm 29.06$ & $49.56 \pm 98$ \\
\hline Rosuvastatin $(\mathrm{n}=50)$ & $306 \pm 46.44$ & $286.4 \pm 11.38$ & $38.56 \pm 4.93$ & $212.90 \pm 35.09$ & $46.99 \pm 74$ \\
\hline Percentage of values & $-39 \%$ & $-41 \%$ & $+9 \%$ & $-63 \%$ & $-55 \%$ \\
\hline
\end{tabular}

TLC - Total cholesterol, TGL -Total triglyceride, HDL -High-density lipoprotein, LDL- low-density lipoprotein, VLDL - very low-density lipoprotein

Table 3: Lipid profile levels among the two groups after the 8 weeks treatment of atorvastatin and rosuvastatin.

\begin{tabular}{|llllll|}
\hline Groups $[\mathrm{n}=100]$ & $\begin{array}{l}\text { Mean TLC } \\
(\mathbf{m g} / \mathbf{d L})\end{array}$ & $\begin{array}{l}\text { Mean TGL } \\
(\mathbf{m g} / \mathbf{d L})\end{array}$ & $\begin{array}{l}\text { Mean HDL } \\
(\mathbf{m g} / \mathbf{d L})\end{array}$ & $\begin{array}{l}\text { Mean LDL } \\
(\mathbf{m g} / \mathbf{d L})\end{array}$ & Mean VLDL(mg/dL) \\
\hline Atorvastatin $(\mathrm{n}=50)$ & $141 \pm 4.5$ & $168.3 \pm 9.6$ & $43.08 \pm 5.15$ & $183.6 \pm 23.90$ & $38.46 \pm 98$ \\
\hline Rosuvastatin $(\mathrm{n}=50)$ & $106 \pm 3.4$ & $122.4 \pm 9.38$ & $47.67 \pm 4.93$ & $163.9 \pm 6.09$ & $41.89 \pm 74$ \\
\hline Percentage of values & $-53 \%$ & $-42 \%$ & $+16 \%$ & $-54 \%$ & $-51 \%$ \\
\hline
\end{tabular}

TLC was found to be more in group A $(141 \pm 4.5)$ when compared to group B (106 \pm 3.4$)$ which was around $53 \%$ (Table 3). Group B showed a significant reduction in TLC level. Mean triglyceride was found to be more in group A

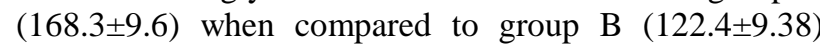
which was around $41 \%$, which is statically significant. Group B showed a significant reduction in TGL level. HDL was found to be more in group B $(47.67 \pm 4.93)$ when compared to group A $(43.08 \pm 5.15)$ which was around $16 \%$ which is statically less significant. Group B showed a significantly increased value in HDL level. LDL was found to be more in group A $(183.6 \pm 23.90)$ when compared to group B (163.9 \pm 6.09$)$ which was around $63 \%$ which is statically significant. Group B showed a significant reduction in LDL level. VLDL was found to be more in group A $(38.46 \pm 98)$ when compared to group B $(41.89 \pm 74)$ which was around $51 \%$ which is statically 
significant. Group B showed a significant reduction in VLDL level.

\section{DISCUSSION}

This study on Asian patients demonstrated an approximately $10 \%$ greater reduction in LDL-C levels with rosuvastatin. This superiority of rosuvastatin is in keeping with the findings of several other trials done on other racial groups. Notable amongst these is the landmark statin therapies for elevated lipid levels compared across doses to rosuvastatin (STELLAR) trial done on 2431 patients comparing rosuvastatin with atorvastatin, simvastatin, and pravastatin. $^{9}$ Across a wide dose range, rosuvastatin produced a significantly greater reduction in LDL-C levels as compared to its competitors. Similarly, Milionis et al. demonstrated a greater LDL-C lowering effect of $10 \mathrm{mg}$ rosuvastatin as compared to that of $20 \mathrm{mg}$ atorvastatin in patients with primary hyperlipidemia. Physicians should remain aware of the doses of different statins while applying the results of this present study to clinical practice. ${ }^{10}$ This is because different statins, with dose adjustment, can be therapeutically equivalent in reducing LDL-C as concluded Wlodarczyk et al, in a meta-analysis. A unique finding of this study is a reduction in HDL-C levels with both statins. Barakat et al, have earlier reported similar phenomenon with rosuvastatin, atorvastatin, and pravastatin. This is in contrast to the well-known fact that statins produce the modest elevations in HDL-C levels. Different statins vary in their HDL-C raising ability and the baseline HDL-C and TG levels are a predictor of statininduced increases in HDL-C. ${ }^{11}$ One possible explanation for these results is a high frequency of diabetics $(75 \%)$ enrolled in this study, as diabetes is known to blunt the HDL response to statins. Poor compliance to treatment cannot be a reason since beneficial effects on LDL-C levels have been seen with both statins. New European guidelines published in 2003 recommend a more stringent target (LDL-C $<2.5 \mathrm{mmol} / \mathrm{L}$ ) than that used when the present study was planned. Further analysis of the 4-week (fixed-dose) LDL-C data indicated that rosuvastatin 10mg treated significantly more patients the new 2003 European goal of $<2.5 \mathrm{mmol} / \mathrm{L}$ than atorvastatin $10 \mathrm{mg}$. As expected, the absolute percentages of patients achieving the more stringent 2003 goal were lower than the absolute percentages achieving the 1998 goal at 4 weeks, but the greater efficacy of rosuvastatin 10mg compared with atorvastatin $10 \mathrm{mg}$ remained the same. ${ }^{12}$ As more clinical trial evidence becomes available regarding the positive effects of intensive lipid-lowering among patients with diabetes, it is likely that even more stringent LDL-C goals will be recommended. Indeed, national cholesterol education program adult treatment panel III recommendations were recently reviewed and a target of LDL-C $<70 \mathrm{mg} / \mathrm{dL} \quad(1.8 \mathrm{mmol} / \mathrm{L})$ was suggested as a therapeutic option for individuals considered to be at very high risk including those with both type 2 diabetes and established cardiovascular disease. Although the statin effect on LDL particle concentration was attenuated in comparison with the effect on LDL cholesterol, the fractional HDL particle concentration response was higher than that of HDL cholesterol. ${ }^{13}$ ApoA-I levels were only modestly increased ( $4-6 \%$ by RSV and $\_1$ to $2 \%$ by ATV), consistent with the known heterogeneity of HDL particles with regard to ApoA-I and lipid content. Thus, the observed treatment effects could reflect an increase in relatively cholesterol-depleted. HDL particles. ${ }^{14}$ Whether this cholesterol depletion is due to higher cholesterol ester transfer protein-mediated reverse cholesterol transport activity or to reduced cholesterol uptake from the periphery uncertain. A recent report described the apparent dysfunction of HDL in patients with the metabolic syndrome thus, any mechanistic hypothesis would not necessarily apply to other patient groups. ${ }^{15}$ Restoring HDL function may be particularly valuable in these patients, but the utility of statins for this purpose remains to be established. However, these studies also suggest that combination of rosuvastatin and atorvastatin was well tolerated and is as safe as therapy with the individual agents used as monotherapy. ${ }^{16}$ These studies also suggest that data up to 2 years support the safety of this combination. Other treatment strategies for normalizing multiple lipid parameters in patients with mixed dyslipidemia include the addition of nicotinic acid or omega 3-fatty acids to statin therapy. Both strategies have resulted in improvements in lipid parameters other than LDL-C. ${ }^{17}$

\section{CONCLUSION}

In conclusion, the present study showed that Rosuvastatin $10 \mathrm{mg} / \mathrm{dl}$ had better response in lipid-profile regulation except for increasing HDL-C levels in IHD patients than Atorvastatin $10 \mathrm{mg} / \mathrm{dl}$. Further study will be needed to determine whether more stringent statin monotherapy or combination treatment, with the goal of further reducing LDL particle concentration, will translate into better outcomes in patients with the metabolic syndrome.

Funding: No funding sources

Conflict of interest: None declared

Ethical approval: The study was approved by the Institutional Ethics Committee

\section{REFERENCES}

1. Athyros VG, Papageorgiou AA, Athyrou VV, Dimitriadis DS, Kontopoulos AG. Atorvastatin and micronized fenofibrate alone and in combination in type 2 diabetes with combined hyperlipidemia. Diabet Care. 2002;25(7):1198-202.

2. Bakker-Arkema RG, Davidson MH, Goldstein RJ, Davignon J, Isaacsohn JL, Weiss SR et al. Efficacy and safety of a new HMG-CoA reductase inhibitor, atorvastatin, in patients with hypertriglyceridemia. JAMA. 1996;275(2):128-33.

3. Cannon CP, Braunwald E, McCabe CH, Rader DJ, Rouleau JL, Belder R, et al. Intensive versus moderate lipid lowering with statins after acute coronary syndromes. N Eng J Med. 2004;350(15):1495-504. 
4. M McKenney JM, Jones PH, Adamczyk MA, Cain VA, Bryzinski BS, Blasetto JW. Comparison of the efficacy of rosuvastatin versus atorvastatin, simvastatin, and pravastatin in achieving lipid goals: results from the STELLAR trial. Curr Med Res Opin. 2003;19(8):689-98.

5. Fiévet C, Staels B. Combination therapy of statins and fibrates in the management of cardiovascular risk. Current opinion in lipidology. 2009;20(6):505.

6. Kwang KK, Michael JQ, Seung HH, Wook-Jin C, Jeong YA, Yield-Hea S, et al. Additive Beneficial Effects of Fenofibrate Combined with Atorvastatin in the Treatment of Combined Hyperlipidemia. Am J Cardiol. 2005;45(10):1649-53.

7. Athyros VG, Papageorgiou AA, Hatzikonstandinou HA, Didangelos TP, Carina MV, Kranitsas DF et al. Safety and efficacy of long-term statin-fibrate combinations in patients with refractory familial combined hyperlipidemia. Am J Cardiol. 1997;80(5):608-13.

8. Blake GJ, Otvos JD, Rifai N, Ridker PM.Low-density lipoprotein particle concentration and size as determined by nuclear magnetic resonance spectroscopy as predictors of cardiovascular disease in women. Circulation. 2002;106:1930-7.

9. Comparison of the efficacy and safety of rosuvastatin versus atorvastatin, simvastatin, and pravastatin across doses (STELLAR Trial). Am J Cardiol. 2003;92:15260 .

10. Hedman M, Matikainen T, Fohr A. Efficacy and safety of pravastatin in children and adolescents with heterozygous familial hypercholesterolemia: A prospective clinical follow-up study. J Clin Endocrinol Metab. 2005;90:1942-52.

11. Cromwell WC, Otvos JD, Keyes MJ, PencinaMJ, Sullivan L, Vasan RS, Wilson PW, et al. LDL particle number and risk of future cardiovascular disease in the Framingham offspring study implications for LDL management. J Clin Lipidol. 2007;1:583-92.

12. Downs JR, Clearfield M, Weis S. Primary prevention of acute coronary events with lovastatin in men and women with average cholesterol levels: results of AFCAPS/TexCAPS. Air Force/Texas Coronary Atherosclerosis Prevention Study. JAMA. 1998;279:1615-22.

13. Fiévet C, Staels B. Combination therapy of statins and fibrates in the management of cardiovascular risk. Current Opinion in Lipidology. 2009;20(6):505-11.

14. Ginsberg HN, Stalenhoef AF. The metabolic syndrome: targeting dyslipidemia to reduce coronary risk. J Cardiovasc Risk. 2003;10:121-8.

15. Judith M.E. drug treatment of hyperlipidemia in women. JAMA. 2004:291(18):2243-52.

16. Kathiresan S, Otvos JD, Sullivan LM, Keyes MJ, Schaefer EJ, Wilson PW, et al. Increased small lowdensity lipoprotein particle number: a prominent feature of the metabolic syndrome in the Framingham Heart Study. Circulation. 2006;113:20-9.

17. Kurtosis DN, Millions H, Bairaktari E, Elisaf MS. Efficacy of a combination of atorvastatin and micronized fenofibrate in the treatment of severely mixed hyperlipidemia. Eur J Clin Pharmacol. 2000;56(9-10):631-5.

Cite this article as: Sivaraj R, Umarani S. Usage of lipid-lowering drugs such as atorvastatin and rosuvastatin in the management of hyperlipidemia in multiple vessel wall block patients. Int J Basic Clin Pharmacol 2018;7:133-7. 